\title{
Both-sided Large Abdominoscrotal Hydrocele Associated with Testicles Atrophy
}

Amir Halilbasic, Nesad Hotic, Fahrija Skokic, Edin Husaric, Emir Rahmanovic, Meliha Halilbasic Department of Paediatric surgery, University Clinical Centre Tuzla, Tuzla, Bosnia and Herzegovina

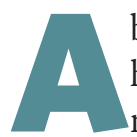
bdominoscrtoal hydrocele is unusual condition with large scrotal hydrocele, which communicates through narrow inguinal channel with abdominal component. Abdominoscrotal hydrocele is not a benign condition, because complications such as acute appendicitis, testicular dismorphism, ureterohydronephrosis, paratesticular malignity have been described earlier. This case study describes one year old boy with both-sided abdominoscrotal hydrocele. Abdominal masses connected with hydrocele need to induce a suspicion of this condition. Early diagnosis and existing surgical techniques for treatment of this disorder are the key factors in prevention of complications associated to this disorder. KEY wORDS: AbDominosCrotal Hydrocele, Abdominal mass. TodDLER-SURgiCAL TREATMENT, COMPLICATIONS.

Corresponding author: Amir Halilbasic, MD. Department of pediatric surgery, UKC Tuzla. Tel.: + 387061259 016 . Fax: + 387035250 47. E-mail: hamir@bih.net.ba

\section{INTRODUCTION}

Abdominoscrotal hydrocele (ASH) is usual condition with large scrotal hydrocele, which communicates through narrow ingvinal channel with abdominal component. This condition is very rare among paediatric population and so far, less than 125 cases have been reported with one-sided abdominoscrotal hydrocele. Both-sided ASH is even rarer and so far, less than 20 cases have been reported among paediatric population $(1,2)$.

It is assumed that ASH occurs by the formation of scrotal hydrocele, under the influence of higher fluid pressure in it, propagating through ingvinal channel into abdominal cavity during the first few months of life (3). Abdominoscrotal hydrocele is not a benign condition, because complications such as acute appendicitis, testicular dismorphysim, ureterohydronephrosis and paratesticular malignity have been described earlier (4).

\section{CASE REPORT}

Healthy eleven months old boy was admitted to Pediatric clinic of the University Clinical Centre due to bothsided large hydrocele. During medical examination, it was found that the boy had both-sided tense hydrocele and tumour masses on both sides in lower parts of abdomen. Parents said that immediately at birth, they could see both-sided hydrocele on their son, which were increasing and becoming tenser as the time was passing. During medical assessment, movement of abdominal masses at pressure to the same sided hydrocele was noticed, which was more evident on the right side (Figure 1).

By ultrasound examination, large cystic anehogenous formations in abdomen, on both sides were found, paravesic, demonstrating transition from cystic abdominal formation into ingvi- nal channel (Figure 2). Computerized tomography (CT) was used to confirm the diagnosis of both-sided abdominoscrotal hydrocele, where both side structures, in the form of sand watch, could be seen at coronal profile (Figure 3).

Surgical treatment included total excision of both-side abdominoscrotal hydrocele through ingvinal approach, where hydrocele was first punctuated to evacuate the content, and then a complete excision of abdominal and scrotal part of hydrocele was done including reconstruction of both ingvinal channels. Abdominal portion of hydrocele extended retroperitoneally and no communication with peritoneal cavity was noted. During operation, right testicle was significantly decreased, morphologically changed, entirely without connection with ductus spermaticus, which ended completely and with no traces of epididymal tissue near the right testicle. The remains of the right testicles are

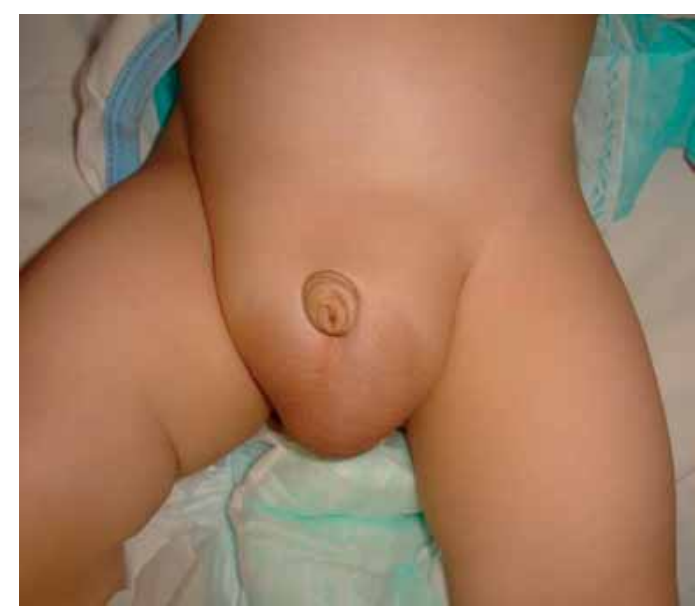

FIGURE 1. Exterior look of a child with both-sided abdominoscrotal hydrocele 


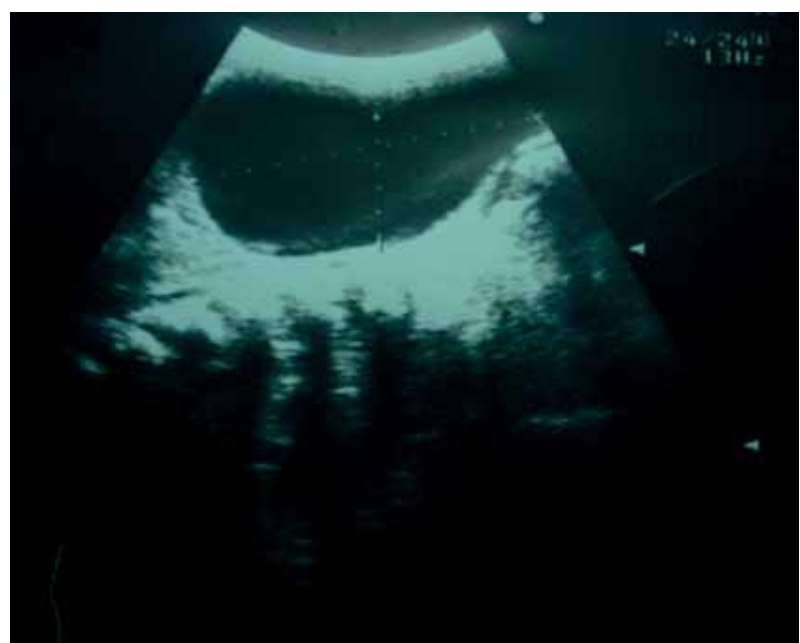

FIGURE 2. Ultrasound assessment of abdominal hydrocele component with transition into ingvinal channel

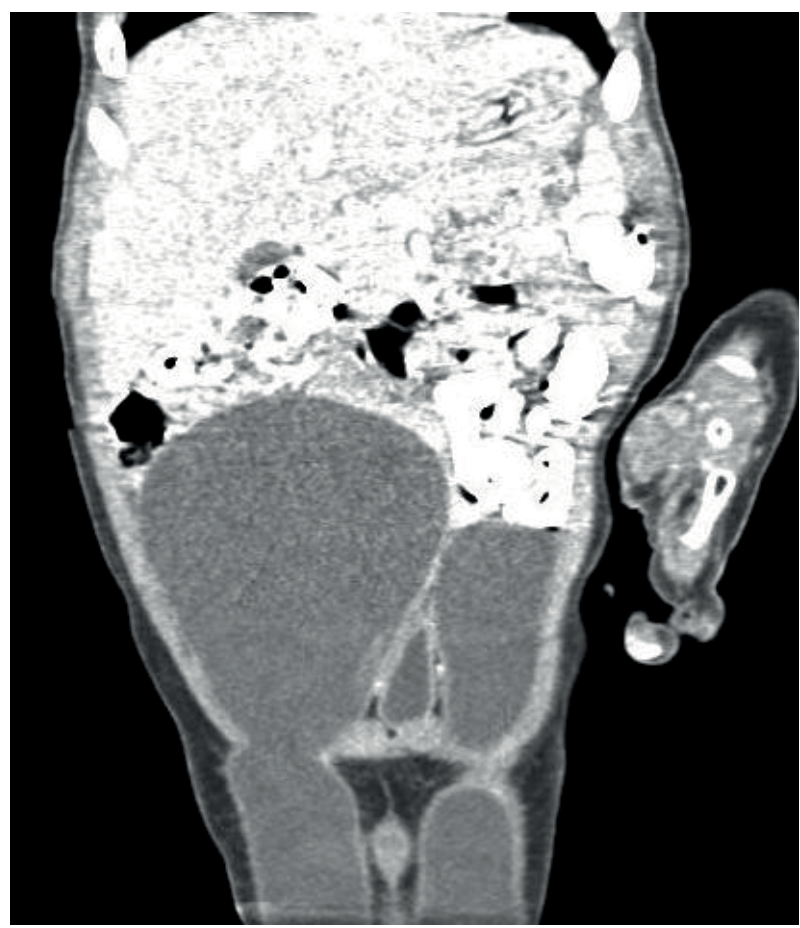

FigURE 3. Coronal CT scan of both-sided abdominoscrrotal hydrocele

removed and sent to histological analysis, which confirmed the testicle atrophy with intestitial fibrosis and a complete lack of epididymal tissue. Based on macroscopic assessment, left testicle was of a proper appearance. Post-operative procedure went smoothly and during the medical control after 6 months, both sides of ingvinoscrotal regions looked normally, without any signs of hydrocele recidives and further development of the left testicle was normal.

\section{DISCUSSION}

Abdominoscrotal hydrocele was described for the first time in 1834 by
Dupuytren, who called it hydrocele en bissac, while Bickle defined this condition in 1919 as abdominoscrotal hydrocele (3). It is assumed that the lesion develops when the procesus vaginalis obliterates along the inside opening of ingvinal channel, which results with fluid accumulation in tunic vaginalis. Increased pressure within hydrocele occurring with fluid accumulation propagates through inside opening of ingvinal channel, which is enabled and guided with non-stretchable walls of ingvinal channel (5). It was published earlier that (6) abdominal part of abdominoskrotal hydrocele may be laid retroperitoneally of preperitoneally, and in our case the abdominal component was laid retroperitoneally. Diagnosis of this lesion should be established by physical assessment and ultrasound check, which should be enough to confirm the abdominoscrotal hydrocele diagnosis. During physical assessment, it was noticed that at pressure to abdominal component, scrotal component increased and vice versa, which was called „springing back ball“ (7). Although some authors include diagnostic processing and magnetic resonance and computerised tomography, these invasive methods should be kept for clear cases after ultra sound check and for critically diseased children (8).

Spontaneous withdrawal of abdominoscrotal hydrocele was described in one case only (9). Considering the complications associated with abdominoscrotal hydrocele such as ureterohydronephrosis (10), acute appendicitis (2), testicular dismorphysm (11) and paratesticular malign mezoteliom (12), surgical treatment as selected treatment is recommended after the establishment of the exact diagnosis (13). Some authors (3) developed and proposed algorithm for treatment of abdominoscrotal hydrocele, which included the option of conservative treatment and monitoring of such children from neonatal period for several months, in expectation of spontaneous resolution. If, during that period, eventual complications are noticed, such children should go to surgical treatment. Most frequent approach used in surgical treatment of abdominoscortal hydrocele is ingvinal approach (3) which we used in case of our patient, though there are reported cases that were treated in scrotal way with less invasive surgical treatment $(1,14,15)$ and laparoscopic methods (16). Selection of surgical technique is individual in terms of the approach to surgical treatment of patient with abdominoskrotal hydrocele. In case of our patient, right testicle was morphologically changed, but histologically, the testicle atrophy with interstitial fibrosis and complete lack of epididymis was confirmed.

The available literature does not report any case of abdominoscrotal hydrocele associated with testicles atrophy and complete lack of epididymis at surgical treatment. So far, morphological testicle disorders have been described, without histological confirmation of intestitial fibrosis. So far, histological analyses of testicle with dismorphosis have shown normal histological structure of testicle tissue (17). In one case, lack of epididymis near testicle was described, but histological analysis confirmed the existence of epididymal tissue incorporated into the hydrocele wall (18).

Most of testicles with dismorphosis achieved in post-operative period a complete recovery (1). In the light of these facts, surgical treatment is preferable type of treatment for patients with abdominoscrotal hydrocele, because the approach is the least invasive, post-operative complications are rare, and eventual consequences associated with abdominoscrotal hydrocele are avoided $(3,15)$. 


\section{CONCLUSION}

Abdominoscrotal hydrocele is a benign disturbance, which sometimes has serious complications. Abdominoscrotal hydrocele should always be considered when there is a patient with scrotal hydrocele and abdominal mass. Detailed monitoring of newborns with tense hydrocele is recommended, because the formation of abdominal component and occurrence of abdominoscrotal hydrocele may be expected in their case. All the patients with abdominoscrotal hydrocele must be processed diagnostically in details, with a focus on eventual complications associated with this condition. Surgical treatment, regardless the technique selected by the surgeon, represents a method of the selection in abdominoscrotal hydrocele treatment and is accompanied by rare complications. Close post-operative monitoring of such patients after the treatment is recommended.

\section{REFERENCES}

1. Cozzi DA, Mele E, Ceccanti S, Pepino D, d'Ambrosio G, Cozzi F. Infantile abdominoscrotal hydrocele: a not so benign condition. J Urol, 2008; 180(6): 2611-5.

2. Yarram SG, Dipietro MA, Graziano K,
Mychaliska GB, Strouse PJ. Bilateral giantabdominoscrotal hydroceles complicated by appendicitis. Pediatr Radiol, 2005; 35(12): 1267-70.

3. Cuervo JL, Ibarra H, Molina M. Abdominoscrotal hydrocele: its particular characteristics. J Pediatr Surg, 2009; 44(9): 1766-70.

4. Blevrakis E, Anyfantakis DI, Sakellaris G. Abdominoscrotal hydrocele in a 9-month old infant. Hernia, 2010 Jan 7. [Epub ahead of print].

5. Brodman HR, Brodman LE, Brodman RF. Etiology of abdominoscrotal hydrocele. Urology, 1977; 10(6): 564-5.

6. Gentile DP, Rabinowitz R, Hulbert WC. Abdominoscrotal hydrocele in infancy. Urology, 1998; 51(5A Suppl): 20-2.

7. Spier LN, Cohen H, Kenigsberg K. Bilateral abdominoscrotal hydrocele: a case report. J Pediatr Surg, 1995; 30(9): 1382-3.

8. Estevão-Costa J, Morgado H, SoaresOliveira M, Campos M, Carvalho JL. Hemorrhagic abdominoscrotal hydrocele. A challenging entity. J Pediatr Surg, 2005; 40(4): 731-3.

9. De Renzo CC, Barone JG. Nonoperative management of abdominoscrotal hydroceles in an infant. Urology, 2006; 68(2): 428.e9-11.

10. Klin B, Efrati Y, Mor A, Vinograd I. Unilateral hydroureteronephrosis caused by abdominoscrotal hydrocele. J Urol, 1992; 148(2 Pt 1): 384-6.

11. Chamberlain SA, Kirsch AJ, Thall EH, Emanuel ER, Hensle TW. Testicular dys- morphism associated with abdominoscrotal hydroceles during infancy. Urology, 1995; 46(6): 881-2.

12. Velasco AL, Ophoven J, Priest JR, Brennom WS. Paratesticular malignant mesothelioma associated with abdominoscrotal hydrocele. J Pediatr Surg, 1988; 23(11): 1065-7.

13. Mahomed AA, Stockdale EJ, Varghese J, Youngson GG. Abdominoscrotal hydrocoeles: little place for conservatism. Pediatr Surg Int, 1998; 13(2-3): 186-8.

14. Belman AB. Abdominoscrotal hydrocele in infancy: a review and presentation of the scrotal approach for correction. J Urol, 2001; 165(1): 225-7.

15. Kajbafzadeh AM, Talab SS, Elmi A, Mahboubi AH, Pourmalek P, Esfahani SA et al. Modified scrotal approach for correction of abdominoscrotal hydrocele in children: clinical presentation and description of technique. Urology, 2010; 76(1): 87-91.

16. Kinoshita Y, Shono T, Nishimoto Y, Masumoto K, Taguchi T, Suita S. A case of an abdominoscrotal hydrocele surgically treated under laparoscopic assistance. J Pediatr Surg, 2006; 41(9): 1610-2.

17. Avolio L, Chiari G, Caputo MA, Bragheri R. Abdominoscrotal hydrocele in childhood: is it really a rare entity? Urology, 2000; 56(6): 1047-9.

18. Park J, Gioia K, Wasnick RJ. Abdominoscrotal hydrocele in infancy with incorporated epididymal tissue. J Pediatr Urol, 2009 Jul 16. [Epub ahead of print]. 